\title{
Creation of the Natural Objects Displacements Fields According to Satellite Data
}

\author{
Aleksey A. Buchnev* and Valeriy P. Pjatkin \\ Institute of Computational Mathematics \\ and Mathematical Geophysics SB RAS \\ 6 Akademika Lavrenteva, Novosibirsk, 630090, Russia
}

Received 03.03.2015, received in revised form 30.04.2015, accepted 19.06.2015

The technology of construction of vector fields of natural objects spatial displacements (ice fields, water masses, cloud formations in the atmosphere) at different times multispectral satellite data is discussed. The technology is based on finding the maxima of mutual correlation coefficient between two neighboring objects in a series of sequential images.

Keywords: mutual correlation, target, vector fields, series of multitemporal images.

DOI: 10.17516/1999-494X-2015-8-6-701-705.

\section{Построение полей перемещений природных объектов по данным космических спутников}

\author{
А.А. Бучнев, В.П. Пяткин \\ Институт вычислительной математики \\ и математической геофизики СО РАН \\ Россия, 630090, Новосибирск, пр. Лаврентьева, 6
}

В статье рассматривается технология построения векторных полей пространственных перемещений природных объектов (ледяных полей, водных масс, облачных образований в атмосфере) по разновременным многоспектральным спутниковым данным. Технология основана на нахождении максимумов коэффициента взаимной корреляции между объектами двух соседних изображений в серии последовательных изображений.

Ключевые слова: взаимная корреляция, эталон, векторное поле, серия разновременных изображений.

Определение пространственных перемещений природных объектов по разновременным спутниковым изображениям основано на нахождении максимумов коэффициента взаимной

(C) Siberian Federal University. All rights reserved

* Corresponding author E-mail address: baa@ooi.sscc.ru 
корреляции между объектами двух соседних изображений в серии последовательных изображений $[1,2]$. В [3] аналогичный подход рассматривается в качестве метода распознавания образов, известного как корреляционное сопоставление. В обоих случаях корреляция используется как средство поиска эквивалентов объекта-эталона, представленного в виде изображения $w(x, y)$ размерами $J \times K$, на изображении $f(x, y)$ размерами $M \times N$, предполагается, что $J \leq M$ и $K \leq N$. Коэффициент взаимной корреляции

$$
\gamma(x, y)=\frac{\sum_{s} \sum_{t}\left[f(x+s, y+t)-f_{m}(x, y)\right]\left[w(s, t)-w_{m}\right]}{\left\{\sum_{s} \sum_{t}\left[f(x+s, y+t)-f_{m}(x, y)\right]^{2} \sum_{s} \sum_{t}\left[w(s, t)-w_{m}\right]^{2}\right\}^{1 / 2}} .
$$

Здесь $w_{m}$ - среднее значение пикселов в эталоне $w, f_{m}$ - среднее значение элементов изображения $f$ в области, покрываемой эталоном.

Для космических изображений вследствие во многом случайного характера представленных на космических снимках изображений интересующих нас природных объектов невозможно говорить о каком-либо предопределенном словаре образов. Такой словарь образов формируется динамически во время анализа серии изображений, и он является индивидуальным для каждого изображения. Построение словаря основано на подходах, изложенных в [4]. Элементы словаря, называемые объектами-эталонами, или просто эталонами, являют собой квадратную область исходного изображения заданного размера. Они отыскиваются в некоторой окрестности узлов квадратной сетки таким образом, чтобы обеспечить максимальное значение управляющего параметра - дисперсии либо энтропии. Если при этом окажется, что достигнутое максимальное значение управляющего параметра превышает заданный порог, то соответствующая часть изображения объявляется эталоном. Другими словами, процедура построения объектов-эталонов направлена на поиск квадратных областей с максимальной «изменчивостью». Для таких областей высока вероятность того, что соответствующий объект не будет полностью разрушен при переходе к следующему изображению серии. Кроме того, снижается вероятность учета случайных корреляций для участков изображений с фоновыми распределениями пикселей изображений. Следующий шаг в определении пространственных перемещений объектов - поиск позиций найденных эталонов на следующем изображении. Смещение определяется для каждого из найденных эталонов. Поиск новой позиции эталона производится внутри квадратной области, центр которой совпадает с исходной позицией эталона. Новой позицией эталона считается позиция, в которой достигается максимальное значение коэффициента взаимной корреляции. В процессе сканирования области поиска эталон может подвергаться преобразованиям масштабирования и поворота. Среди всех отобранных позиций могут быть позиции, удовлетворяющие пороговым условиям - минимально допустимое значение коэффициента корреляции и минимально допустимое смещение. Процесс может быть продолжен итеративно для следующих изображений серии: найденные области с максимальным значением корреляции объявляются эталонами, к ним могут быть добавлены эталоны, полученные с помощью описанной ранее процедуры поиска эталонов, для нового набора эталонов находятся их позиции на следующем изображении и т.д. Описанная процедура построения полей перемещений природных объектов практически без изменений используется 
для анализа перемещений облачных образований на основе серии последовательных изображений, полученных с геостационарных спутников. Эти изображения выходят со сравнительно небольшим временным интервалом, вследствие чего можно ожидать высокой корреляции между соседними изображениями.

Единственное дополнение - редактирование векторных полей, которое заключается в удалении явно ошибочных векторов (такая ситуация есть следствие того, что облака на разных высотах могут иметь разные направления перемещений).

Приведенные рисунки демонстрируют фрагмент полученного векторного поля перемещения облачных образований в атмосфере. Демонстрируется процесс перемещения вихря. Векторное поле построено по пяти последовательным изображениям с ИСЗ METEOSAT-8. Приведен первый кадр последовательности изображений (рис. 1), последний кадр (рис. 2) и соответствующее векторное поле (рис. 3).

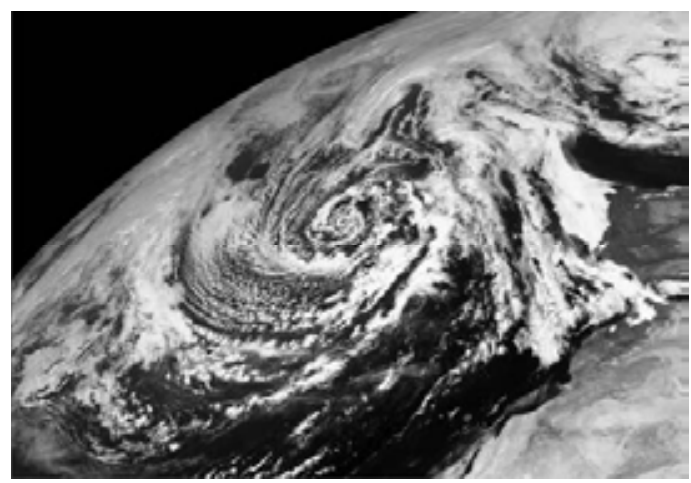

Рис. 1

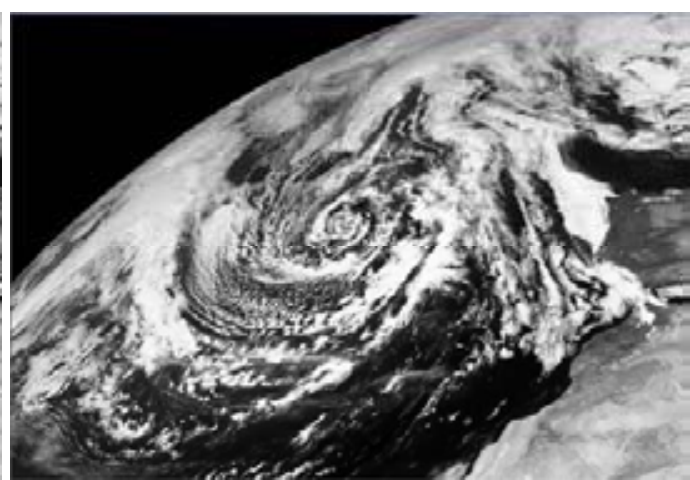

Рис. 2

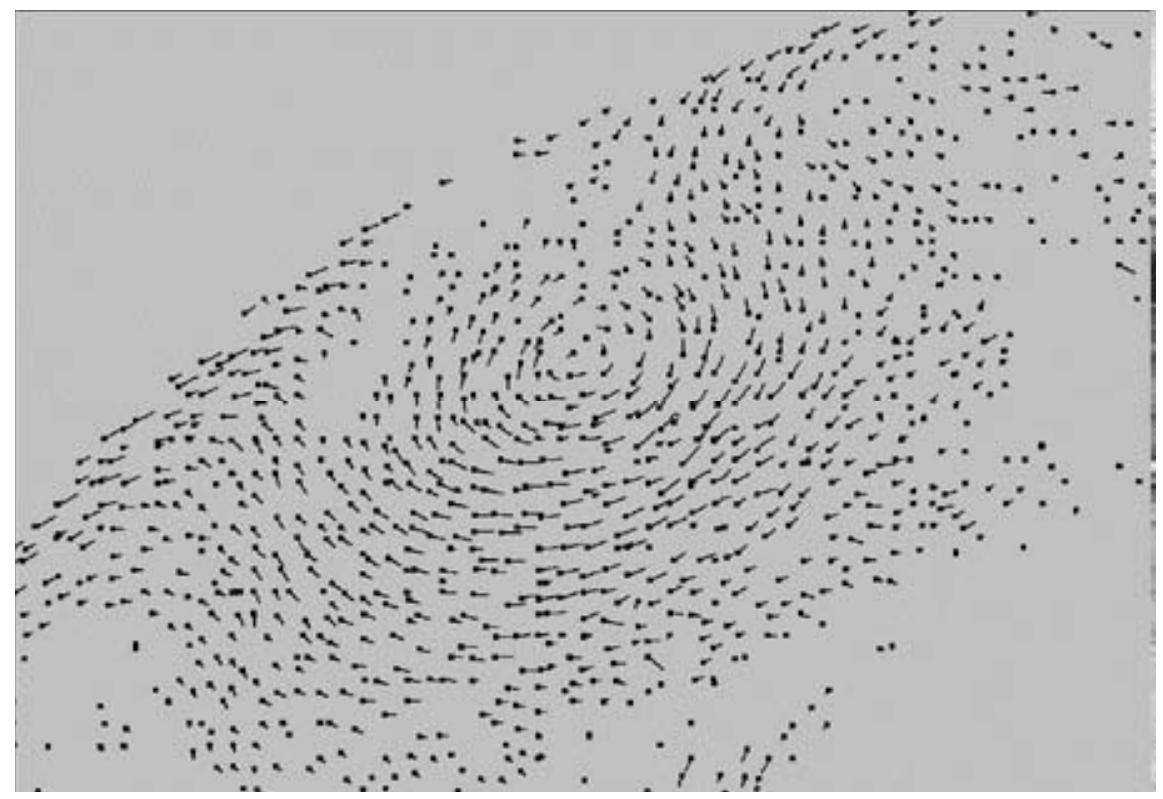

Рис. 3 
Однако для водных масс и ледяных полей изображения в серии расположены по времени далеко друг от друга (в частности, изображения ледяных полей являются результатом объединения (построения мозаик) радиолокационных изображений, полученных в течение суток). По этой причине, во избежание явно ошибочных корреляций, необходимо в максимально возможной степени ужесточать требования как к построению эталонов, так и к уровню корреляции. В результате полученного количества векторов смещений не хватает для построения векторного поля, характеризующего динамику процесса. В этой связи для данных природных объектов предложено и реализовано следующее дополнение к технологии построения векторных полей перемещений. На основе позиций найденных эталонов строится триангуляция Делоне, которая вместе с соответствующими координатами новых позиций эталонов на следующем изображении определяет семейство кусочно-аффинных преобразований плоскости. На исходном изображении строится квадратная сетка заданного размера, и к каждому узлу сетки, попадающему внутрь выпуклой оболочки позиций эталонов, применяется аффинное преобразование, определяемое треугольником, в котором находится этот узел. Между исходными и новыми позициями узлов сетки строятся отрезки, определяя тем самым поле перемещений. На рис. 4, любезно предоставленном ФГБУ «НИЦ «Планета» (г. Москва), демонстрируется результат применения описанной технологии к построению полей

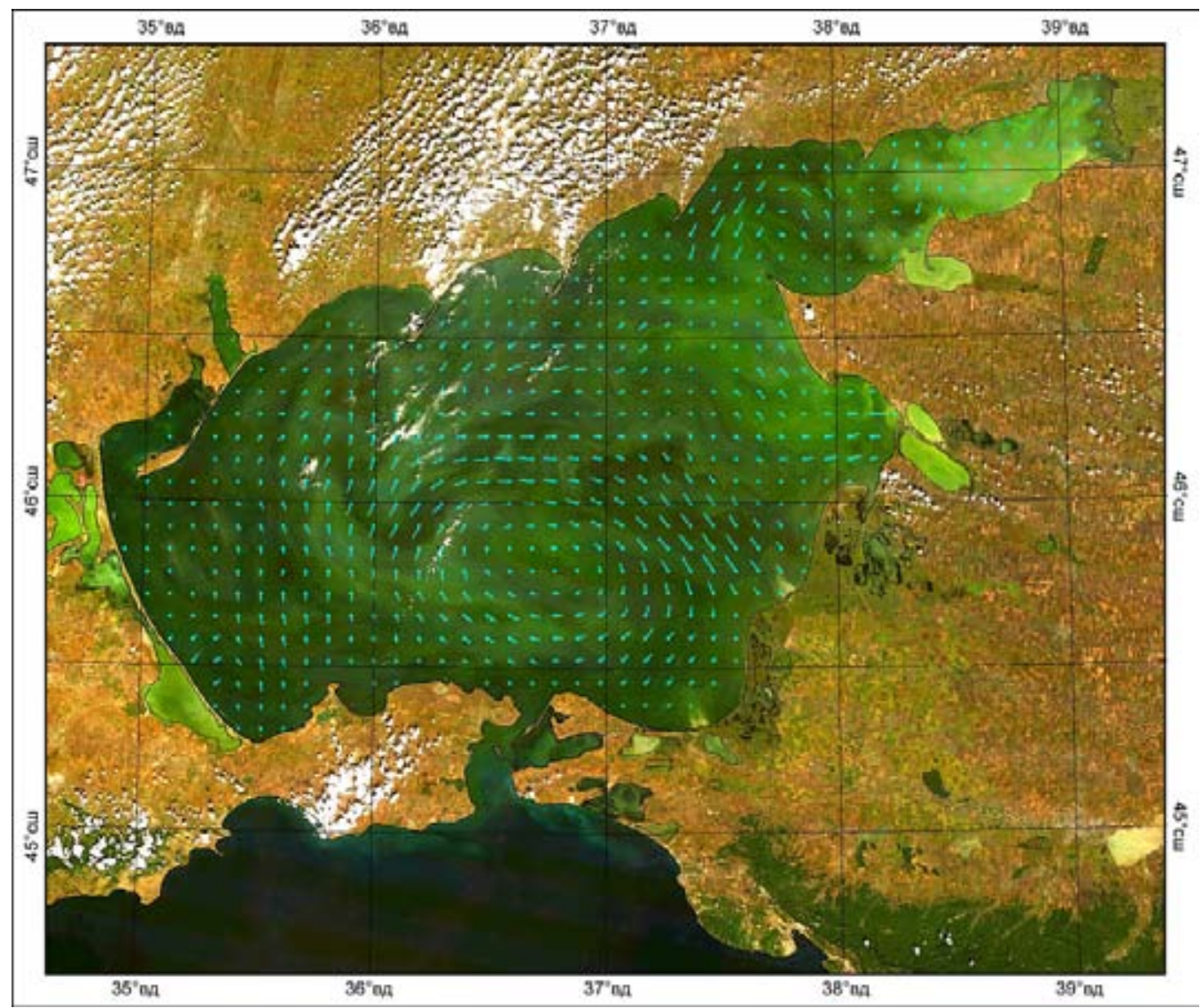

Рис. 4 
переноса водных масс в акватории Азовского моря по изображениям, полученным с ИСЗ Terra (EOS AM-1), сканер Modis.

Программные технологии включают в себя функции получения статистических характеристик построенных векторных полей - распределение векторов по направлениям (с задаваемой дискретностью изменения угла) и распределение скоростей векторов.

Описанные программные технологии для определения пространственных перемещений природных объектов по разновременным многоспектральным космическим изображениям созданы в ходе выполнения совместных работ с ФГБУ «НИЦ «Планета» в рамках Федеральной целевой программы: «Федеральная космическая программа России на 2006-2015 годы». Соответствующий программный комплекс внедрён в практику оперативной и научной работы центра. Комплекс активно используется в космическом мониторинге полярных регионов Земли (ледяные поля), российского сектора Чёрного и Азовского морей (водные массы) и облачных образований в атмосфере.

Работа выполнена частично при финансовой поддержке Российского фонда фундаментальных исследований (проект № 13-07-00068) и Программы № 43 Президиума РАН (проект № 32).

\section{Список литературы}

[1]. Asmus V.V., Buchnev A.A., Pyatkin V.P., Salov G.I. // Pattern Recognition and Image Analysis. 2009. Vol. 19. № 3. P. 372.

[2]. Бучнев А.А., Пяткин В.П. // Автометрия. 2009. Т. 45. № 5. С. 40.

[3]. Гонсалес Р., Вудс Р. Цифровая обработка изображений: пер. с англ. / ред. П.А. Чочиа. М.: Техносфера, 2012. 1103 c.

[4]. MSG Meteorological Products Extraction Facility. Algorithm Specification Document. // Doc. No. EUM/MSG/SPE/022. June 2004. Issue 2.6. 1. 